\title{
Supporting Treatment decision making to Optimise the Prevention of STROKE in Atrial Fibrillation: The STOP STROKE in AF study. Protocol for a cluster randomised controlled trial
}

\author{
Melina Gattellari ${ }^{1,2}$, John M Worthington ${ }^{1,3,4^{*}}$, Dominic Y Leung ${ }^{3,5}$ and Nicholas Zwar ${ }^{2}$
}

\begin{abstract}
Background: Suboptimal uptake of anticoagulation for stroke prevention in atrial fibrillation has persisted for over 20 years, despite high-level evidence demonstrating its effectiveness in reducing the risk of fatal and disabling stroke.

Methods: The STOP STROKE in AF study is a national, cluster randomised controlled trial designed to improve the uptake of anticoagulation in primary care. General practitioners from around Australia enrolling in this 'distance education' program are mailed written educational materials, followed by an academic detailing session delivered via telephone by a medical peer, during which participants discuss patient de-identified cases. General practitioners are then randomised to receive written specialist feedback about the patient de-identified cases either before or after completing a three-month posttest audit. Specialist feedback is designed to provide participants with support and confidence to prescribe anticoagulation. The primary outcome is the proportion of patients with atrial fibrillation receiving oral anticoagulation at the time of the posttest audit.
\end{abstract}

Discussion: The STOP STROKE in AF study aims to evaluate a feasible intervention via distance education to prevent avoidable stroke due to atrial fibrillation. It provides a systematic test of augmenting academic detailing with expert feedback about patient management.

Trial registration: Australian Clinical Trials Registry Registration Number: ACTRN12611000076976.

Keywords: Atrial fibrillation, General practice, Anticoagulation, Decisional support

\section{Background}

Atrial fibrillation (AF) is the most common heart arrhythmia with a prevalence of around $5 \%$ in those aged over 65 , increasing to $10 \%$ in those older than 75 years [1]. AF confers the highest risk for ischaemic stroke of all traditional risk factors, including congestive heart failure, diabetes, and hypertension [2]. In Australia, at least one in four patients admitted to hospital with an ischaemic stroke has AF [3]. Mortality in stroke patients with $\mathrm{AF}$ is around twice the rate in those without it; by

\footnotetext{
* Correspondence: john.worthington@sswahs.nsw.gov.au

'Ingham Institute, Liverpool, Australia

${ }^{3}$ South Western Sydney Clinical School, The University of New South Wales, Sydney, Australia

Full list of author information is available at the end of the article
}

12 months, $40 \%$ of ischaemic stroke patients with AF will have died [3]. Moreover, survivors of AF-related stroke spend a significantly longer time in hospital [3].

Meta-analyses of randomised controlled trials of antithrombotic medication demonstrate that, in the average AF patient with a $5 \%$ chance of an ischaemic stroke each year, oral anticoagulation with adjusted-dose warfarin reduces this annual risk by $67 \%$, in relative terms, to approximately $1.7 \%$ [4]. In contrast, antiplatelet medication, such as aspirin, reduces the risk by only $21 \%$, to approximately $4 \%$ [4]. Despite over 20 years of evidence supporting lifelong anticoagulation as the treatment of choice for most patients with AF [4-6], 50\%$60 \%$ of patients do not receive anticoagulants even if they are at high risk of stroke [7,8]. An Australian study 
of 218 consecutively admitted patients with AF reported that only $20 \%$ were using warfarin on admission [9], although inclusion of an unspecified number of newly diagnosed AF cases would have influenced the reported proportion of under use [9]. In a Canadian stroke unit, 90\% of admitted ischaemic stroke patients with AF were not receiving anticoagulation or were insufficiently anticoagulated at the time of their stroke [10]. In one Australian stroke unit, $68 \%$ of ischaemic stroke patients with known AF before admission either were not anticoagulated or were sub-optimally anticoagulated at the time of their stroke [11]. This persistent evidence-practice gap contributes to the burden of preventable stroke.

Clinicians are often reluctant to prescribe anticoagulation for AF, particularly if patients are elderly or have a heightened risk of falls or other perceived risk of major bleeding [12-19]. However, many patients considered unsuitable for anticoagulation also have an elevated risk of ischaemic stroke, and the risk-benefit trade-off favours anticoagulation in the majority of patients with AF [20-24].

Few studies have evaluated implementation strategies specifically designed to increase the uptake of oral anticoagulation for AF. McAlister et al. [25] reported an increase in antithrombotic prescribing at three months in general practice patients receiving a decision aid. The difference was not sustained at 12 months after randomisation. In a nonrandomised study of Australian primary healthcare physicians in one geographical region of Tasmania, guideline dissemination followed by academic-detailing visits significantly increased prescribing rates of adjusted-dose warfarin compared to a control region [26]. Two other studies in the primary healthcare setting addressed several cardiovascular risk factors, without focusing specifically on AF management [27,28]. Ornstein et al. [27] tested the effect of computerised guidelines, reminder systems, and audit and feedback on prescribing behaviour in managing several cardiovascular risk factors. Posttest differences between intervention and control groups were not statistically significant. Another trial found that locally adapted guidelines, an educational seminar delivered by local opinion leaders, educational materials, and an offer of an educational outreach visit did not increase primary healthcare physicians' adherence to prescribing guidelines for patients with a history of transient ischaemic attack (TIA) or AF [28]. The outcome measure in this study did not distinguish between anticoagulant and antiplatelet prescribing.

Other randomised evaluations of implementation strategies have been carried out with stroke survivors to optimise secondary prevention [29-33]. These have included few AF patients $(n=6$ to $n=99)$ [29-33], and of the two studies reporting outcomes for AF patients, neither found statistically significant improvements in anticoagulant prescribing [32,33].
Specialist decisional support for general practitioners (GPs) merits further investigation. A recent evaluation of a shared-care model for stroke survivors involved liaison between GPs and stroke specialists, facilitated via a nurse specialist. The study found significant improvements in the management of secondary stroke risk and, for AF patients, a nonsignificant improvement in anticoagulation prescribing attributable to the intervention [32]. However, the total number of AF patients in this study was small $(n=32)$. While the multifaceted nature of the study intervention precludes the identification of effective components, it suggests that linking GPs with stroke specialists could enhance clinical decision making. An observational study also reported a significant effect of collaborative involvement between specialists and GPs on appropriate anticoagulation prescribing, providing further support for the hypothesis that specialist input may be effective in improving the management of AF patients [34].

In another study, known as DESPATCH, we are evaluating the effect of expert decisional support to promote the uptake of anticoagulation using feedback from clinical experts in stroke medicine [35]. However, in the DESPATCH study, decisional support is embedded within a multifaceted intervention including written educational materials, three telephone academic-detailing sessions, and a workshop/seminar. We have designed the STOP STROKE in AF study to systematically assess the impact of expert decisional support on the management of AF in a national study.

\section{Methods}

Please see attached file (Additional file 1) for the study flow diagram.

\section{GP recruitment}

We obtained contact details of GPs from a commercially available database [36], which sources information from professional colleges, medical directories, and state medical boards that register all practising clinicians in Australia. After an initial pilot with 100 randomly selected GPs to determine the likely participation rate, we undertook recruitment in two phases, obtaining lists of 3,000 randomly selected GPs from across Australia in 2010 and 2,501 in 2011 (with those selected in 2010 removed from the sampling frame). GPs who were participating in another implementation trial about AF we were carrying out [37] were excluded from the randomly selected samples ( $\mathrm{n}=10$ in 2010 and $\mathrm{n}=13$ in 2011).

GPs were mailed a brief introductory letter inviting them to complete a registration form, to be returned by fax or an enclosed business reply paid envelope, advising us of their interest in an education program about stroke prevention. During the first (i.e., in 2010) and second 
phases of recruitment (i.e., in 2011), we carried out two randomised trials of response-aiding strategies, both of which received ethics approval as amendments to the main study protocol. During the first phase, a statistician independent of the research team randomised 2,250 GPs into three equal-sized groups ( $\mathrm{n}=750$ for each group). The first group was mailed the introductory letter and registration form in an institutional $110 \mathrm{~mm}$ by $220 \mathrm{~mm}$-sized envelope (DL-sized) with the university logo and address printed in black and white on the top left-hand corner. The second group was mailed the letter and form in the institutional envelope and also received complimentary copies of resources provided free of charge by the National Stroke Foundation (NSF) of Australia. The third group was also mailed the NSF resources with their letter and form but received these via an Australia Post "Express Post" envelope representing "priority mail". The remaining 740 GPs were not included in this trial and were mailed the introductory letter and registration form at a different time point to those selected to test response-aiding strategies. During the second wave of recruitment, 2,488 GPs were randomly divided to receive the cover letter and registration form printed on pale yellow $(n=1,247)$ or white paper $(n=1,240)$. Letters were mailed using the institutional DL-sized envelopes with logo and address printed in black and white. This trial was separately registered (ACTRN12611000259943).

GPs who returned their registration forms were mailed an information sheet and a consent form, advising that consenting GPs would be invited to participate in an educational session delivered by a medically trained peer via telephone, after which they would be randomised to receive specialist feedback about the de-identified patient cases (see description of intervention below). GPs who returned a signed consent form by fax or enclosed reply paid envelope were then mailed educational materials in advance of a telephone educational session to be delivered by medical peers. Only those GPs who complete the telephone educational session, providing pretest data, are considered recruited and eligible for randomisation. During the initial stages of the first recruitment phase (i.e., in 2010), GPs returning their registration form were telephoned in advance of receiving the information sheet and consent form, and those GPs not returning a consent form within a minimum of two weeks were mailed a second copy. However, due to resource constraints, this follow-up protocol was abandoned.

\section{Academic detailing}

Enrolled GPs receive printed education materials (see Additional file 2) prior to being contacted by a medically trained peer who carried out a standardised telephone educational session covering key issues concerning the management of AF (see Additional file 3). Topics include the epidemiology of AF [1-3], risk stratification using the validated tools [37], and information about the benefits and risks of antithrombotic medications used to manage $\mathrm{AF}$, including adjusted-dose warfarin and antiplatelet medication, such as aspirin and/or clopidogrel [38-44]. During 2011, the information was revised to include information about a revised risk stratification scheme [45] and dabigatran [46-48], a newly available fixed-dose anticoagulant that received regulatory approval in Australia for stroke prophylaxis in AF in May 2011.

GPs were advised in advance of the telephone educational session to identify patients with AF who are over the age of 65 and not receiving anticoagulation or for whom anticoagulation management had presented difficulties. GPs enrolled during the first phase of recruitment (i.e., in 2010) were advised to select around three or four patients for discussion with the medical peer, while those recruited during the second phase (i.e., from 2011 onwards) were advised to select a minimum of five patients.

At the conclusion of the telephone educational session, the medically trained peer proceeded through a standardised patient pro forma, collecting de-identified information about patient demographics, stroke risk factors, other comorbidities, current antithrombotic medications, and issues relevant to anticoagulation use. Medically trained peers calculated the patient's $\mathrm{CHADS}_{2}$ score with participating GPs, providing general feedback based on evidence-based guideline recommendations, according to patients' $\mathrm{CHADS}_{2}$ scores [24]. The $\mathrm{CHADS}_{2}$ score assigns two points for prior stroke or TIA and one point each for the following risk factors: congestive heart failure, history of hypertension, age over 75, and diabetes. Key safety messages about stroke risk in paroxysmal AF, hypertension, and use of antithrombotic treatment in patients with a history of spontaneous intracranial haemorrhage were also communicated. From late 2011, medical peers also communicated a safety alert issued by the peak Australian drug regulatory body, the Therapeutics Goods Administration (TGA), about the risks of using dabigatran, a newly available oral anticoagulant, in the elderly and in patients with renal impairment [49].

\section{Pretest data collection}

Before commencing the academic-detailing session, medical peers noted GPs' gender and asked participants to report the number of doctors in their practice and whether they were practising full-time or part-time. Medical peers asked GPs whether they had any of the following resources available to assist them in managing AF patients receiving warfarin: a nurse to monitor and recall patients when needed; automated or computerised reminders for noting when patients need to have their 
International Normalised Ratios INR levels tested; point-of-care INR testing; pathology service anticoagulation management; or hospital clinical anticoagulation management, practice-based register, or utilisation of a formal Australian government program whereby pharmacists visit patients to monitor compliance with medications. A modified version of the Provider Decision Process Assessment Instrument [50] measuring GP levels of decisional conflict regarding warfarin use was administered. GPs were asked to indicate their level of agreement (agree, disagree, neither) to five statements ("Whether or not to prescribe warfarin is a difficult decision to make, 'For most patients it is clear that warfarin is the best treatment,' 'It is often difficult to decide if the benefits of warfarin outweigh the risks, or vice versa, 'Generally, patients fully appreciate the benefits of warfarin', 'Generally, patients fully appreciate the risks of warfarin'). Finally, medical peers asked GPs whether they had participated in formal educational activities about AF in the previous 12 months.

The de-identified patient pro forma document contributed to the pretest patient-level data for this study. Information on patient age and sex was first elicited. GPs were then asked to clarify whether the patient had paroxysmal or chronic AF (a response of unsure was permitted) and whether the patient had underlying valvular heart disease or nonvalvular AF. Medical peers asked GPs to indicate if the patient had thyrotoxicosis (yes/no/unsure). Relevant medical history ascertaining stroke risk factors to enable calculation of the $\mathrm{CHADS}_{2}$ score was elicited. History of myocardial infarction, peripheral artery disease, aortic plaque, coronary artery disease, and high cholesterol/hyperlipidemia was also noted, as was current smoking status.

Relevant comorbidities reflecting actual or perceived contraindications to anticoagulation were also elicited. These included the following: falls history (without and without injury), a history of multiple falls, anaemia, recent or current upper gastrointestinal (GI) bleeding within the last month, history of upper GI bleeding, history of lower GI bleeding, history of GI bleeding (location not specified), a treated cause of GI bleeding, cerebral haemorrhage, hepatic insufficiency/moderate to severe liver disease, impaired kidney function, chronic dialysis, renal implantation, abnormal serum creatinine (>200 ųmol/L), hepatic derangement on biochemical testing, excessive alcohol intake (defined as eight or more units per week), coagulopathy, thrombocytopaenia, dementia or cognitive impairment (with and without supervised care), insertion of coronary artery stent (drug-eluting, bare metal, or stent of unknown type), and nonadherence with medication or management. Comorbidities assessing bleeding risk as described above were derived from a standardised scheme [51]. GP participants could nominate other comorbidities they considered relevant for specialist feedback. The pro forma elicited information on current antithrombotic medications; previous use of anticoagulation with adjusted-dose warfarin; and, where relevant, reasons for not using warfarin, for ceasing warfarin, and adverse events whilst receiving warfarin (minor bleeding not requiring hospitalisation, major blood loss requiring hospitalisation, intracranial or intracerebral haemorrhage, including subarachnoid haemorrhage; ischaemic stroke; TIA or amaurosis fugax; sub-therapeutic INR levels; supra-therapeutic INR levels; consistently unstable INR levels). Rate- or rhythm-control medications, nonsteroidal anti-inflammatory drugs (NSAIDs) including COX-2 inhibitors, paracetamol, alternative medications, and other over-the-counter medications in current use were also noted. From 2011 onwards, GPs were asked to note, where relevant, adverse events whilst receiving anticoagulants other than warfarin (most likely dabigatran).

\section{Randomisation}

A statistician external to the study group is carrying out randomisation after participants complete academicdetailing sessions. Randomisation is being stratified by the number of cases GPs identify ( $\leq 2$ or $>3$ ). Results of randomisation are communicated to the study coordinator (MG) who assigns GPs to their respective groups. The statistician involved in the analysis of the trial will receive the randomisation schedule directly from the statistician carrying out randomisation. Block randomisation is being used to control for date of entry into the trial. Block size will be disclosed to the study team only during the write-up of results.

\section{Expert decisional support}

Medically trained peers reminded GPs that they will be randomised to receive specialist feedback about patient de-identified cases either prior to or after posttest evaluation. They were advised to consider this component of the intervention as an educational exercise and, therefore, should not have expectations of timely feedback and should seek any urgently required advice through their usual practices. Data from the de-identified pro forma documents were mailed to MG, who entered the data and summarised information into a one- to twopage document that was forwarded to experts in either neurology, cardiology and stroke. The experts provided written information that was then mailed to GPs randomised to receive expert decisional support prior to the posttest phase of the study (see Additional file 4 for a hypothetical example). Our model for delivering this aspect of the intervention was informed by an intervention developed by McAlister et al., 2006 [52]. 


\section{Waiting list control}

GPs randomised to the waiting list control group were mailed a summary of patient de-identified information, identical to the summary received by GPs randomised to the intervention arm. These GPs also received a statement summarising patient ischaemic stroke risk based on the patient's $\mathrm{CHADS}_{2}$ score. Therefore, patient summaries were identical between groups, with the exception of specialist written feedback.

\section{Posttest data collection}

Posttest data collection is scheduled for approximately 12 weeks after GPs have received either the expert decisional feedback or, for those randomised to the waiting list control group, the patient de-identified summary document. GPs receive another copy of the patient de-identified summary sheet (without expert feedback) attached to a brief posttest questionnaire. For each patient, GPs are asked to indicate current antithrombotic treatment from a list of available choices (warfarin, aspirin, clopidogrel, aspirin and clopidogrel, dipyridamole, dipyridamole with aspirin, dabigatran, other antithrombotic for GP to specify, or no antithrombotic) and, if currently receiving warfarin, to report the last six INR test results and corresponding dates of blood collection. In response to a checklist of items, GPs are asked to specify reasons for any given patient not receiving anticoagulation (patient refusal/reluctance, history of traumatic brain haemorrhage, history of spontaneous brain haemorrhage, history of lower GI bleed, history of upper GI bleed, falls history without injury, falls history with injury, history of GI location not specified, age, cognitive impairment, inadequate supervision at home, patient nonadherence with medication, patient nonadherence with monitoring, specialist doesn't recommend anticoagulation, patient at low risk of stroke, awaiting government subsidisation of fixed-dose anticoagulation with dabigatran, or other reason which GPs are asked to specify).

Whether or not patients are receiving anticoagulation, GPs complete an adapted version of the Provider Decision Process Assessment Instrument [50], consisting of six items assessing GP levels of decisional conflict regarding anticoagulation with adjusted-dose warfarin, with an additional question asking GPs to indicate their level of satisfaction with the current treatment. From 2011 onwards, the posttest evaluation included an additional six questions assessing levels of decisional conflict for a newly available fixed-dose anticoagulant, dabigatran. Posttest data will be entered by personnel blinded to group allocation.

\section{Primary outcome}

The primary outcome is defined as the proportion of patients with AF who, at posttest, are reported by GPs to be receiving anticoagulation.

\section{Secondary endpoints \\ Appropriate antithrombotic treatment according to stroke risk}

In accordance with evidence-based guidelines [38-40], we will use baseline $\mathrm{CHADS}_{2}$ scores in those patients aged over 65 years to judge appropriateness of antithrombotic management. Specifically, patients over 65 years of age with a baseline $\mathrm{CHADS}_{2}$ score of 0 will be considered to be managed appropriately if receiving aspirin, while those with a baseline $\mathrm{CHADS}_{2}$ score of 2 or more will be considered to be receiving appropriate antithrombotic treatment if receiving anticoagulation (either with warfarin or dabigatran). Either anticoagulation or aspirin will be considered appropriate in those with a baseline $\mathrm{CHADS}_{2}$ score of 1 . The expected few cases of patients younger than 65 years of age will be excluded from this analysis. However, a sensitivity analysis will be carried out applying the $\mathrm{CHADS}_{2}$ score to patients younger than 65 years of age, as above, in recognition of inclusion criteria used in recent trials of antithrombotic treatments that included patients younger than 65 where AF was associated with $\mathrm{CHADS}_{2}$ stroke risk factors [4-6].

\section{Appropriate antithrombotic treatment according to stroke risk incorporating quality-control criteria}

In those patients receiving anticoagulation with warfarin, we will only consider anticoagulation to be appropriate for this outcome if GPs, when reporting the six most recent INR results, report an INR result at least monthly and where at least four of the six test results (or twothirds of results in cases where fewer than six are reported) are within therapeutic range (i.e., within 2.03.0). These criteria for appropriate anticoagulation are based on local guidelines [44] and a consistently achieved standard of 'time in therapeutic range' reported in clinical trials [4-6], including one trial of general practice patients with an average age of 81 years [6].

\section{Further refinement of outcome definition}

Combinations of antiplatelet therapies with or without anticoagulation may diverge from standard guideline recommendations but may nonetheless be appropriate in the context of aspirin intolerance, patients with a history of coronary stenting, or in those affected by acute coronary syndromes [42,43,53-55]. We will accept other antiplatelet treatments, such as clopidogrel, dypridamole, and combinations of these treatments, where aspirin would have been considered the evidence-based treatment. Where anticoagulation is considered the evidencebased choice, we will not distinguish between cases where anticoagulation is used alone or in combination with other antithrombotics. 


\section{Decisional conflict}

Scores comprising the seven-item decisional conflict scale, based on a previously validated measure [50] adapted for use in our earlier work and in other studies $[13,14]$, will be summed to produce a total score, whereby higher scores indicate higher levels of decisional conflict (range $=7$ to 35). At the time of writing, warfarin remains the only anticoagulant treatment that is available to all Australian residents via Australia's universal health insurance scheme ensuring government subsidisation of medication. The fixed-dose anticoagulant dabigatran has received regulatory approval for AF but is not currently subsidised by the Australian government. Consequently, the uptake of dabigatran is not expected to be high, and we will restrict this outcome to items assessing decisional conflict about warfarin only, unless uptake of dabigatran is found to be common.

\section{Sensitivity analyses}

In cases that are lost to follow-up (for example, where GPs do not return their posttest audit questionnaires), we will reanalyse our primary and secondary outcomes relating to prescribing behaviour, firstly, assuming no change in antithrombotic management from baseline to posttest and secondly, assuming the desired outcome was not achieved.

\section{Subgroup analyses}

We will carry out subgroup analyses for our primary and secondary outcomes according to the following variables: (a) baseline $\mathrm{CHADS}_{2}$ scores $(0,1$, or $\geq 2$ ); (b) patient age $(<65$ years, $65-74$ years, $75-84$ years, $\geq 85$ years); (c) baseline anticoagulation use (yes versus no); (d) patient sex.

\section{Pretest comparisons between groups}

We will compare groups on the numbers of patients selected by GPs, patient sex, age (mean and median differences and on categorical groupings $<65$ years, $65-74$, $75-84, \geq 85$ years), $\mathrm{CHADS}_{2}$ scores $(0,1, \geq 2$, and mean scores), and use of oral anticoagulation (current at pretest, previous use, or never used).

\section{Sample size estimate}

Our study was powered to detect a $15 \%$ difference in anticoagulation rates between groups. We assumed a baseline use of anticoagulation of $50 \%$ to produce anticonservative (i.e., larger) estimates of the required sample size. In the absence of a design effect, whereby the sample size would be adjusted for clustering of patients by GP, we required GPs to identify 170 patients per group or 340 in total [56]. As patient cases were clustered by GP, we adjusted our sample size estimate by the design effect $D_{\text {eff }}=1+(m-1) \rho$ [57], where $m$ equals the average cluster size and $\rho$ equals the intraclass correlation coefficient. We have selected a value of $m$ to produce anticonservative (i.e., larger) estimates of the required GP sample, as the number of GPs needed will have a greater effect on study resources and its feasibility than the numbers of patient cases identified by GPs. Smaller values of $m$ will produce a sample size estimate requiring greater numbers of GPs. Assuming an average cluster size of three and selecting an intraclass correlation coefficient of 0.029 , informed by results from a previous study [27], our anticipated design effect was 1.06 (i.e., $1+(3-1) \times 0.029)$, producing a required total sample size of $1.06 \times 340=361$. Assuming $20 \%$ of posttest audit questionnaires are not returned by GPs, we require data on 452 de-identified patient cases or 226 per group. If GPs each select three patient cases on average, then we will need to recruit 76 GPs per group (i.e., 226/3), or 152 in total.

\section{Statistical analysis}

Outcomes will be analysed according to the intentionto-treat principle, whereby patients are analysed according to the arm to which their GP cluster was allocated. Clustering will be accounted for at the practice level [57]. A $p$ value of $<.05$ will be used to determine statistical significance of results. Analyses will be carried out by a statistician blinded to group allocation.

\section{Ethics approval}

The Human Research Ethics Committee of the University of New South Wales has approved the study (UNSW HREC Reference Number 07067).

\section{Discussion}

Long-term anticoagulation for AF remains the treatment of choice to prevent stroke in most people with AF. The STOP STROKE in AF study has an active 'control'. Information is delivered via academic detailing with tailored feedback about risk stratification and guideline recommendations. The addition of expert decisional support in our intervention arms allows for a direct test of this component on GP self-reported patient management. The national geographical reach of the study and feasible delivery of the intervention, via telephone and mail, are innovations of the study.

Until recently, warfarin has been the only oral anticoagulant available for managing AF. Two fixed-dose anticoagulants, dabigatran [46,47] and rivoroxaban [58], have received approval for stroke prophylaxis in AF by the US Federal Drug Administration (FDA). Apixaban [59], another fixed-dose anticoagulant, is due to be reviewed by the FDA in 2012. It is unclear whether the advent of new drugs will improve anticoagulation rates. In practice, uptake of dabigatran, the first FDA-approved 
fixed dose oral anticoagulant, has been described as 'disappointing, with an estimated $10 \%$ of AF patients using the drug in the United States [60]. The cost of the drug, the need for twice-daily dosing, a short shelf-life, the lack of an antidote to reverse acute bleeding, the inability to determine the anticoagulation effects of dabigatran, and the increased risk of GI bleeding, dyspepsia, discontinuation, and possibly myocardial infarction, when compared with warfarin, may limit its broader uptake [58]. Recent warnings from regulators in Australia [48] and the United States [61] have emphasised safety concerns about dabigatran, particularly in those with renal impairment and in elderly patients aged over 75 years. In Australia, AF became an approved indication for dabigatran by the local government regulator, the TGA, after which the company manufacturing the drug offered doctors free medication for a limited period of time, or until the drug became subsidised by the Australian government (whichever occurred first). The Australian government has thus far declined the company's application to subsidise the drug. Federal agencies in Australia are currently undertaking a further review of the costs and benefits of the new drug and defining the circumstances in which warfarin may be more cost effective. Other fixed-dose anticoagulants that may come to market in the near future, specifically rivaroxaban and apixaban, share some drawbacks of dabigatran; all three lack an antidote to reverse acute bleeding, and apixaban also requires twice-daily dosing.

Prescribing behaviour seems difficult to shift. The South London secondary prevention programme, also called Stop Stroke, enrolled 523 stroke survivors, 99 of whom had AF. Patients were randomised to receive 'keeping well plans' summarising risk factors and management strategies, including evidence-based prescribing for managing blood pressure, diabetes, and AF, that were updated over a 12-month period [33]. The intervention had no effect on any of the study outcomes, including anticoagulant prescribing for AF patients.

Clinician fears about the side effects of anticoagulation appear to have greater influence on anticoagulant prescribing than do concerns about stroke risk. Clinicians are less likely to prescribe anticoagulation in patients with $\mathrm{AF}$ if one of their AF patients receiving anticoagulation is hospitalised for a major bleed, yet prescribing behaviour is not influenced if untreated AF patients are hospitalised for an ischaemic stroke [62]. In our national Australian survey $[13,14]$, GPs with prior experiences of a haemorrhagic stroke in AF patients report a heightened sense of responsibility for that outcome. In contrast, GPs with untreated AF patients who had a stroke were no more likely to feel responsible for this outcome than other GPs. Further, our survey showed that GPs appeared overly cautious in prescribing anticoagulation in the presence of any perceived risk of bleeding, even where treatment benefits clearly outweighed the risk of harm. Other Australian surveys show that GPs are reluctant to prescribe anticoagulation for nonvalvular atrial fibrillation in the elderly or in the presence of perceived bleeding risks, which would not contraindicate anticoagulation according to available evidence [15-17].

The STOP STROKE in AF study aims to redress clinician wariness and concerns over the use of anticoagulation and support effective decision making to build practitioner confidence and increase the appropriate uptake of anticoagulation in AF.

In addition to our own research, we are aware of one other Australian study evaluating a risk assessment tool in general practice for improving the management of $\mathrm{AF}$ $[63,64]$. It is hoped that these studies will collectively identify effective implementation strategies to better inform GPs on AF management, closing the evidencepractice gap in Australia and elsewhere.

\section{Progress}

Required numbers of GPs have been recruited. We expect posttest data collection to be completed in 2012 . Results are expected in 2013.

\section{Additional files}

\section{Additional file 1: Consort flow diagram.}

Additional file 2: Summary of written information mailed to all GPs.

Additional file 3: Content summary of academic detailing session. Additional file 4: Example of patient summary and expert feedback.

\section{Competing interests}

The authors state they have no competing interests to declare.

\section{Acknowledgements}

Randomisation for the STOP STROKE in AF is being performed by Patrick McElduff, Clinical Research Design, IT and Statistical Support Unit (CReDITSS), University of Newcastle. Chris Goumas, Data Analyst, School of Public Health and Community Medicine, The University of New South Wales, and Anthony Yeo, Biostatistician, Ingham Institute, carried out randomisation for the response-aiding strategies for the first and second phase of recruitment, respectively. The STOP STROKE in AF trial is supported by the BUPA Foundation, Australia. MG was supported by an NHMRC Public Health PostDoctoral Training Fellowship (\#301136) at the time of writing the submission for project funding and was supported by a Commonwealth Department of Health and Ageing, Primary Health Care Research, Evaluation and Development Mid-Level Career Research Fellowship from September 2007 to September 2011. The opinions expressed in this publication do not necessarily reflect those of the Commonwealth of Australia, which does not accept any liability for loss, damage, or injury incurred by the use of or reliance on the information contained herein. The funding bodies had no role in the design of the study, the writing of this manuscript, or in the decision to submit the manuscript for publication. We thank the GPs who have participated in the study and gratefully acknowledge The National Stroke Foundation of Australia for providing free resources for use in our trial of response-aiding strategies. The STOP STROKE in AF content experts were Associate Professor John M Worthington and Professor Dominic Leung. The STOP STROKE in AF specialist expert committee included Associate Professor 
David Blacker, Dr Emma Blackwood, Professor Christopher Bladin, Associate Professor Brian Chambers, Associate Professor Dennis Cordato, Dr Andy Evans, Professor Dominic Leung, Associate Professor Stephen Read, Professor David Schultz, and Associate Professor John M Worthington. The STOP STROKE in AF academic detailers/medical peers were Drs. Jemima Grant, Kate Johnston, Amelia Lem, Declan Mulvaney, Jay Ramanathan, Brian Simmons, and Anna Windsor. The project management team consisted of Melina Gattellari, Laura Morgan-Deller (past), and Catherine Johnson (past).

\section{Author details}

${ }^{1}$ Ingham Institute, Liverpool, Australia. ${ }^{2}$ School of Public Health and Community Medicine, The University of New South Wales, Sydney, Australia. ${ }^{3}$ South Western Sydney Clinical School, The University of New South Wales, Sydney, Australia. ${ }^{4}$ Department of Neurology, Liverpool Health Service, South Western Sydney Local Health Network, Liverpool, Australia. ${ }^{5}$ Department of Cardiology, Liverpool Health Service, South Western Sydney Local Health Network, Liverpool, Australia.

\section{Authors' contributions}

JMW initiated the group's interest in developing an intervention in general practice to reduce stroke risk in patients with atrial fibrillation. MG is the lead investigator on the study. MG and JMW had major input into study design and conceptualisation and cowrote the protocol for publication. NZ and DYL provided input into study protocol at the time of its conceptualisation. JMW and DYL led the expert decisional-support component of the intervention and informed outcome assessment. JMW prepared authored information resources. All authors reviewed and provided feedback on the protocol. All authors have read and approved the final submission.

Received: 9 May 2012 Accepted: 6 July 2012

Published: 6 July 2012

\section{References}

1. Lake FR, Cullen KJ, de Klerk NH, McCall MG, Rosman DL: Atrial fibrillation and mortality in an elderly population. ANZ Med 1989, 19:321-326.

2. Wolf PA, Abbot RD, Kannel WB: Atrial fibrillation as an independent risk factor for stroke: the Framingham study. Stroke 1991, 22:983-988.

3. Gattellari M, Goumas C, Aitken R, Worthington JM: Outcomes for patients with ischaemic stroke and atrial fibrillation: The PRISM study (A Program of Research Informing Stroke Management). Cerebrovasc Dis 2011, 32:370-382.

4. Hart RG, Pearce LA, Aguilar MI: Meta-analysis: antithrombotic therapy to prevent stroke in patients who have nonvalvular atrial fibrillation. Ann Intern Med 2007, 146:857-867.

5. Hart RG, Pearce LA, Aguilar MI: Adjusted-dose warfarin versus aspirin for preventing stroke in patients with atrial fibrillation (author' reply). Ann Intern Med 2007, 147:591

6. Mant J, Hobbs R, Fletcher K, Roalfe A, Fitzmaurice D, Lip GYH: Warfarin versus aspirin for stroke prevention in an elderly community population with atrial fibrillation (the Birmingham Atrial Fibrillation Treatment of the Aged Study, BAFTA): A randomised controlled trial. Lancet 2007, 370:493-503.

7. Olgilvie IM, Welner SA, Cowell W, Lip GY: Characterization of the proportion of untreated and antiplatelet therapy treated patients with atrial fibrillation. Am J Cardiol 2011, 108:151-61

8. Olgilvie IM, Newton N, Welner SA, Cowell W, Lip GYH: Underuse of oral anticoagulants in atrial fibrillation. A systematic review. Am J Med 2010, 123:638-645.

9. Bajorek BV, Krass I, Ogle SJ, Duguid MJ, Shenfield GM: Optimizing the use of antithrombotic therapy for atrial fibrillation in older people: a pharmacist-led multidisciplinary intervention. J Am Geriatric Soc 2005, 53:1912-20

10. Gladstone DJ, Bui E, Fang J, Laupacis A, Lindsay MP, Tu JV, Silver FL, Kapral MK. Potentially preventable strokes in high-risk patients with atrial fibrillation who are not adequately anticoagulated. Stroke 2009, 40:235-240.

11. Ahmad O, Ahmad KE, Dear KBG, Harvey I, Hughes A, Lueck CJ: Atrial fibrillation and anticoagulation in a stroke unit population. Internal Medicine Journal 2009, 39:752-56.
12. Pugh D, Pugh J, Mead GE: Attitudes of physicians regarding anticoagulation for atrial fibrillation: a systematic review. Age and Ageing 2011, 40:675-83

13. Gattellari M, Worthington J, Zwar N, Middleton S: Barriers to the use of anticoagulation for non-valvular atrial fibrillation (NVAF): a representative survey of Australian family physicians. Stroke 2008, 39:227-230.

14. Gattellari M, Worthington JM, Zwar NA, Middleton S: The management of non-valvular atrial fibrillation (NVAF) in Australian general practice: bridging the evidence-practice gap. A national, representative postal survey. BMC Fam Pract 2008, 9:62.

15. Shen A, Cordato D, Ng J, Hung WT, Kokkinos J, Karr M, Yin Chan DK: Anticoagulant usage for primary stroke prevention: a general practitioner survey in local areas of metropolitan Sydney. J Clin Neurosci 2008, 15:166-71.

16. Peterson GM, Boom K, Jackson SL, Vial JH: Doctors' beliefs on the use of antithrombotic therapy in atrial fibrillation: identifying barriers to stroke prevention. Internal Medicine Journal 2002, 32:15-23.

17. Arendts G, Krishnaraj M, Paull G, Rees D: Management of atrial fibrillation in the acute setting: findings from an Australasian survey. Heart, Lung \& Circulation 2010, 19:423-7.

18. Zimetbaum PJ, Thosani A, Yu HT, Xiong Y, Lin J, Kothawala P, Emons M: Are atrial fibrillation patients receiving warfarin in accordance with stroke risk? Am J Med 2010, 123:446-53.

19. Mazzaglia G, Filippi A, Alacqua M, Cowell W, Shakespeare A, Mantovani LG, Bianchi C, Cricelli C: A national survey of the management of atrial fibrillation with antithrombotic drugs in Italian primary care. Thromb Haemost 2010, 103:968-75.

20. Poli D, Antonucci E, Testa S, Tosetto A, Ageno W, Palareti G, Italian Federation of Anticoagulation Clinics: Bleeding risk in very old patients on vitamin $\mathrm{K}$ antagonist treatment: results of a prospective collaborative study on elderly patients followed by Italian Centres for Anticoagulation. Circulation 2011, 124:769-71.

21. Singer DE, Chang Y, Fang MC, Borowsky LH, Pomernacki NK, Udaltsova N, Go AS: The net clinical benefit of warfarin: anticoagulation in atrial fibrillation. Ann Intern Med 2009, 151:297-305.

22. Garwood CL, Corbett TL: Use of anticoagulation in elderly patients with atrial fibrillation who are at risk for falls. Annals of Phamacotherapy 2008, 42:523-32.

23. Man-Son-Hing M, Nichol G, Lau A, Laupacis A: Choosing antithrombotic therapy for elderly patients with atrial fibrillation who are at risk for falls. Arch Intern Med 1999, 159:677-85.

24. Gage BF, Birman-Deych E, Kerzner R, Radford MJ, Nilasena DS, Rich MW: Incidence of intracranial hemorrhage in patients with atrial fibrillation who are prone to fall. Am J Med 2005, 118:612-7.

25. McAlister FA, Man-Son-Hing M, Straus SE, Ghali WA, Anderson D, Majumdar PG, Cox JL, Fradette M, for the Decision Aid in Atrial Fibrillation (DAAFI) Investigators: Impact of a patient decision aid on care among patients with nonvalvular atrial fibrillation: a cluster randomized trial. CMAJ 2005 173:496-501.

26. Jackson SL, Peterson GM, Vial JH: A community-based educational intervention to improve antithrombotic drug use in atrial fibrillation. Ann Pharmacother 2004, 38:1794-1799.

27. Ornstein $\mathrm{S}$, et al: A multimethod quality improvement intervention to improve preventive cardiovascular care: a cluster randomized trial. Ann Intern Med 2004, 141:523-32.

28. Wright J, Bibby J, Eastham J, Harrison S, McGeorge M, Patterson C, Price N, Russell D, Russel I, Small N, Walsh M, Young J: Multifaceted implementation of stroke prevention guidelines in primary care: cluster-randomised evaluation of clinical and cost effectiveness. Qual Saf Health Care 2007, 16:51-59.

29. Allen KR, Hazelett S, Jarjoura D, Wickstrom GC, Hua K, Weinhardt J, Wright K: Effectiveness of a postdischarge care management model for stroke and transient ischemic attack: a randomized trial. Journal of Stroke and Cerebrovascular Disease 2001, 11:88-98.

30. Banet GA, Felchlia MA: The potential utility of a shared medical record in a 'first-time' stroke population. J Vasc Nurs 1997, 15:29-35.

31. Ellis $G$, Rodger J, McAlpine C, Langhorne P: The impact of stroke nurse specialist input on risk factor modification: a randomised controlled trial. Age Ageing. 2005, 34:389-392.

32. Joubert J, Reid C, Barton D, Cumming T, McLean A, Joubert L, Barlow J, Ames D, Davis S: Integrated care improves risk-factor modification after 
stroke: initial results of the Integrated Care for the Reduction of Secondary Stroke model. J Neurol Neurosurg Psychiatry 2009, 80:279-284.

33. Wolfe CDA, Redfern J, Rudd AG, Grieve AP, Heuschmann PU, McKevitt C: Cluster randomized controlled trial of a patient and general practitioner intervention to improve the management of multiple risk factors after stroke. Stop Stroke. Stroke. 2010, 41:2470-2476.

34. Choudhry NK, Soumerai SB, Normand SLT, Ross Degnan D, Laupacis A, Anderson GM: Warfarin prescribing in atrial fibrillation: the impact of physician, patient and hospital characteristics. Am J Med 2006, 119:607-615.

35. Gattellari M, Leung DY, Ukoumunne OC, Zwar N, Grimshaw J, Worthington JM: Study protocol: The DESPATCH study: Delivering stroke prevention for patients with atrial fibrillation- a cluster cluster randomised controlled trial. Implement Sci 2011, 6:48.

36. AMPCo Direct. http://www.ampcodirect.com.au/DynamicPages.asp? cid=11\&navid=2 last accessed January2, 2012.

37. Gage BF, Waterman AD, Shannon W, Boechler M, Rich MW, Radford MJ: Validation of clinical classification schemes for predicting stroke: results from the National Registry of Atrial Fibrillation. JAMA 2001, 285:2864-70.

38. Hankey GJ, on behalf of the National Blood Pressure Advisory Committee of the National Heart Foundation: Non-valvular atrial fibrillation and stroke prevention Position Statement. MJA 2001, 174:234-239.

39. Fuster V, Ryden LE, Cannom DS, Crijns HJ, Curtis AB, Ellenbogen KA, Halperin JL, Kay GN, Le Huezey JY, Lowe JE, Olsson SB, Prystowsky EN, Tamargo JL, Wann LS, Smith SC Jr, Priori SG, Estes NA, Ezekowitz MD, Jackman WM, January CT, Lowe JE, Page RL, Slotwiner DJ, Stevenson WG, Tracy CM, Jacobs AK, Anderson JL, Albert N, Buller CE, Creager MA, Ettinger SM, Guyton RA, Halperin JL, Hochman JS, Kushner FG, Ohman EM, Stevenson WG, Tarkington LG, Yancy CW, American College of Cardiology Foundation/American Heart Association Task Force: 2011 ACCF/AHA/HRS focused updates incorporated into the ACC/AHA/ESC 2006 guidelines for the management of patients with atrial fibrillation: a report of the American College of Cardiology Foundation/American Heart Association Task Force on practice guidelines. Circulation 2011 , 123:e269-367.

40. Albers GW, Amarenco P, Easton JD, Sacco RL, Teal P, American College of Chest Physicians: Antithrombotic and thrombolytic therapy for ischemic stroke: American College of Chest Physicians Evidence-based Clinical Practice Guidelines (8 ${ }^{\text {th }}$ Edition). Chest 2008, 13:630S-669S.

41. Baker RI, Coughlin PB, Gallus AS, Harper PL, Salem HH, Wood EM: Position statement. Wafarin reversal: consensus guidelines, on behalf of the Australasian society of thrombosis and haemostasis. MJA 2004, 181:492-497.

42. The ACTIVE Investigators: Effect of clopidogrel added to aspirin in patients with atrial fibrillation. N Eng J Med 2009, 360:2066-2078.

43. ACTIVE Writing Group of the ACTIVE Investigators, Connolly S, Pogue J, Hart R, Pfeffer M, Hohnloser S, Chrolavicius S, Pfeffer M, Hohnloser S, Yusuf S: Clopidogrel plus aspirin versus oral anticoagulation for atrial fibrillation in the Atrial Fibrillation Clopidogrel Trial With Irbesartan for Prevention of Vascular Events (ACTIVE W): a randomized controlled trial. Lancet 2006, 367:1903-1912.

44. Gallus AS, Baker RI, Chong BH, Ockelford PA, Street AM, On behalf of the Australasian Society of Thrombosis and Haemostatis: Position statement. Consensus guidelines for warfarin therapy. Recommendations from the Australasian Society of Thrombosis and Haemostatis. Medical Journal of Australia 2000, 172:600-605.

45. Lip GYH, Nieuwlaat R, Pisters R, Lane DA, Crijns HJGM: Refining clinical risk stratification for predicting stroke and thromboembolism in atrial fibrillation using a novel risk factor based approach: the Euro Heart Survey on atrial fibrillation. Chest 2010, 137:263-272.

46. Connolly SJ, Ezekowitz MD, Yusuf S, Eikelboom J, Oldgren J, Parekh A Pogue J, Reilly PA, Themeles E, Varrone J, Wang S, Alings M, Xavier D, Zhu J, Diaz R, Lewis BS, Darious J, Deiner HC, Joyner CD, Wallentin L: RE-LY Steering Committee and Investigators. Dabigatra versus warfain in patients with atrial fibrillation. N Engl J Med 2009, 361:1139-51.

47. Connolly SJ, Ezekowitz MD, Yusyf S, Reilly PA, Wallentin L, for the Randomised Evaluation of Long-term Anticoagulation Therapy Investigators: Newly identified events in the RE-LY trial. N Engl J Med 2010, 363:1875-1876.

48. National Prescribing Service: Dabigatran (Pradaxa). NPS Radar Review. http:// www.nps.org.au/news_and_media/media_releases/repository/ Dabigatran_Pradaxa_NPS_RADAR_Review. Last accessed January 2, 2012
49. Therapeutic Goods Administration. http://www.tga.gov.au/safety/alerts-medicinedabigatran-111005.htm Last accessed Janaury 2, 2012.

50. Dolan JG: Method for evaluating health care providers' decision making: the Provider Decision Process Assessment Instrument. Medical Decision Making 1991, 19:38-41

51. Lip GYH, Frison L, Halperin JL, Lane DA: Comparative validation of a novel risk score for predicting bleeding risk in anticoagulated patients with atrial fibrillation: the HAS-BLED (Hypertension, Abnormal Renal/Liver Function, Stroke, Bleeding History or Predisposition Labile INR, Elderly, Drugs/Alcohol Concomitantly) score. J Am Coll Cardiol 2011, 57:173-80.

52. McAlister FA, Fradette M, Graham M, Majumdar SR, Ghali WA, Williams R, Tsuyuki RT, McMeekin J, Grimshaw J, Knudtson ML: A randomized trial to assess the impact of opinion leader endorsed evidence summaries on the use of secondary prevention strategies in patients with coronary artery disease: The ESP-CAD trial protocol (NCT00175240). Implement SCi 2006, 1:11

53. Paiken JS, Mehta SR, Eikelboom JW: Role of triple antithrombotic therapy in patients with atrial fibrillation and coronary artery stents. Medical Journal of Australia 2010, 192:487-488.

54. Hansen ML, Sorensen R, Clausen MT, Fog-Petersen ML, Raunso J, Gadsboll N, Gislason GH, Folke F, Andersen SS, Schramm TK, Abildstrom SZ, Poulsen HE, Kober L, Torp-Pedersen C: Risk of bleeding with single, dual, or triple therapy with warfarin, aspirin, and clopidogrel in patients with atrial fibrillation. Arch Intern Med 2010, 170:1433-41.

55. Rubboli A, Halperin JL, Airaksinen KE, Buerke M, Eeckhout E, Freedman SB, Gershlick AH, Schlitt A, Tse HF, Verheugt FW, Lip GY: Antithrombotic therapy in patients treated with oral anticoagulation undergoing coronary artery stenting. An expert consensus document with focus on atrial fibrillation. Annals of Medicine 2008, 40:428-36.

56. Dupont WD, Plummer WD: Power and sample size calculations: a review and computer program. Control Clin Trials 1990, 11:116-28.

57. Murray DM: Design \& analysis of group randomized trials. NY: Oxford University Press; 1998.

58. Patel MR, Maheffrey KW, Garg J, Pan G, Singer DE, Hacke W, Breithardt G, Halperin JL, Hankey GJ, Piccini JP, Becker RC, Nessel CC, Paolini JF, Berkowitz SD, Fox KA, Califf RM: Rivaroxaban versus warfarin in nonvalvular atrial fibrillation. N Engl J Med 2011, 365:883-91.

59. Granger CB, Alexander JH, McMurray JJ, Lopes RD, Hylek EM, Hanna M, AlKhalidi HR, Ansell J, Atar D, Avezum A, Bahit MC, Diaz R, Easton JD, Ezekowitz JA, Flaker G, Garcia D, Geraldes M, Gersh BJ, Golitsyn S, Goto S, Hermosillo AG, Hohnloser SH, Horowitz J, Mohan P, Jansky P, Lewis BS, Lopez-Sendon JL, Pais P, Parkhomenko A, Verheugt FW, Zhu J, Wallentin L, ARISTOTLE Committees and Investigators: Apixaban versus warfarin in patients with atrial fibrillation. New England Journal of Medicine 2011, 365:981-92.

60. Zoller ML: Dabigatran's first A-Fib year starts warfarin's decline. eCardiology News. http://www.ecardiologynews.com/news/arrhythmiaselectrophysiology/single-article/dabigatran-s-first-a-fib-year-starts-warfarin-sdecline/446ecf58b7.html November 13, 2011. Last accessed January 2, 2012.

61. Federal Drugs Administration: Federal Drugs Administration. http://www.fda.gov/ Safety/MedWatch/Safetylnformation/SafetyAlertsforHumanMedicalProducts/ ucm282820.htm Last accessed January 2, 2012.

62. Choudhry NK, Anderson GM, Laupacis A, Ross-Degnan D, Normant SLT, Soumerai SB: Impact of adverse events on prescribing warfarin in patients with atrial fibrillation: matched pair analysis. BMJ 2006, 332:141-145.

63. Bajorek BV, Masood N, Krass I: Optimising the utilisation of antithromnotic therapy for stroke prevention in AF, Development and evaluation of a computerised antithrombotic risk assessment took (CARAT), Poster presentation $20^{\text {th }}$ European Stroke Conference. Hamburg, Germany: May 24-27, 2011. http://www.esc-archive.eu/hamburg11/Ongoing\%20Trials \%20ESC\%20Hamburg\%202011.pdf (Last accessed April 15 2012).

64. Bajorek BV, Masood N, Krass I: Development of a computerised antithrombotic risk assessment tool (CARAT) to optimise therapy in older persons with atrial fibrillation. Australasian Journal on Ageing 2012, 31:102-09.

doi:10.1186/1748-5908-7-63

Cite this article as: Gattellari et al: Supporting Treatment decision making to Optimise the Prevention of STROKE in Atrial Fibrillation: The STOP STROKE in AF study. Protocol for a cluster randomised controlled trial. Implementation Science 2012 7:63. 